\title{
Prolonged Mechanical Ventilation After Aortic Arch Repair Requiring Deep Hypothermic Circulatory Arrest: Incidence, Effect on Outcome, and Clinical Predictors
}

\author{
John G. Augoustides ${ }^{*}, 1$, Wilson Szeto ${ }^{2}$, Benjamin A. Kohl ${ }^{1}$, Doreen Cowie ${ }^{3}$, Aaron Hoo ${ }^{1}$, \\ Andrew J. Gambone ${ }^{1}$ and David R. Jobes ${ }^{1}$ \\ ${ }^{I}$ Department of Anesthesiology and Critical Care, Cardiothoracic and Vascular Section, Hospital of the University of \\ Pennsylvania, Philadelphia, Pennsylvania, USA \\ ${ }^{2}$ Department of Surgery, Division of Cardiothoracic Surgery, Hospital of the University of Pennsylvania, Philadelphia, \\ Pennsylvania, USA \\ ${ }^{3}$ Department of Clinical Perfusion, Hospital of the University of Pennsylvania, Philadelphia, Pennsylvania, USA
}

\begin{abstract}
Objective: To delineate the incidence, outcome impact, and clinical predictors of prolonged mechanical ventilation (PMV) after adult aortic arch repair requiring deep hypothermic circulatory arrest (AAR-DHCA)

Aims: (1) To determine the incidence of PMV after AAR-DHCA. (2) To determine whether PMV after AAR-DHCA is a multivariate predictor for mortality or length of stay in the intensive care unit. (3) To determine multivariate predictors for PMV after AAR-DHCA. (4) To determine whether aprotinin influences PMV after AAR-DHCA.

Study Design: Retrospective and observational. Prolonged mechanical ventilation was defined as mechanical ventilation via an endotracheal tube for longer than 72 hours.

Study Setting: Single large university hospital.

Participants: All adults undergoing AAR-DHCA in 2000 and 2001.

Main Results: Cohort size was 144. Antifibrinolytic exposure was 100\%: aprotinin 66\% and aminocaproic acid 34\%. The incidence of AF was $21.5 \%$. PMV did not independently predict for mortality or prolonged stay in the intensive care unit. The multivariate predictors for PMV were chronic obstructive pulmonary disease, stroke, and infection. In multivariate analysis, aprotinin exposure has no significant association with PMV.

Conclusions: PMV after AAR-DHCA is common, but does not independently predict mortality or ICU stay. The risk of PMV after AAR-DHCA increases with preexisting chronic obstructive pulmonary disease, stroke and infection. Perioperative intervention should focus on protection against stroke and infection.
\end{abstract}

\section{INTRODUCTION}

Pulmonary complications after aortic arch repair with deep hypothermic circulatory arrest (AAR-DHCA) are common [1-3]. Prolonged mechanical ventilation (PMV) in this setting has a published incidence of up to $27 \%$ and significantly prolongs length of stay [1-3]. Although PMV after AAR - DHCA is common and important, its clinical predictors have not been fully described. Furthermore, the clinical studies of this perioperative complication have not accounted for aprotinin exposure, an antifibrinolytic agent in AARDHCA that has recently been associated with improved pulmonary outcome [4]. In the light of the above considerations, this clinical study was undertaken at the Hospital of the University of Pennsylvania with the following aims:

(1) To determine the incidence of respiratory complications (including PMV) after AAR-DHCA;

*Address correspondence to this author at the Cardiothoracic Anesthesia and Intensive Care, Department of Anesthesiology and Critical Care, 680 Dulles, Hospital of the University of Pennsylvania, 3400 Spruce Street, Philadelphia, PA 19104-4283, USA; Tel: (215) 662-7631; Fax: (215) 3498133; E-mail: yiandoc@hotmail.com
(2) To determine whether PMV after AAR - DHCA is a multivariate predictor for mortality or prolonged stay in the intensive care unit (ICU);

(3) To determine multivariate predictors for PMV after AAR - DHCA;

(4) To determine whether aprotinin exposure influences PMV after AAR - DHCA.

\section{METHODS}

Data Collection: After approval from the institutional review board, all adults undergoing AAR - DHCA from January $1^{\text {st }} 2000$ to December $31^{\text {st }} 2001$ were studied. Perioperative data sets (demographics; anesthetic and surgical descriptors; clinical outcomes) were abstracted from the medical records and subsequently electronically archived. (Microsoft Access, Microsoft, Seattle, WA, USA).

\section{OUTCOME DEFINITIONS}

(1) Pulmonary: Chronic obstructive disease (COPD) was defined by any two of the following criteria: preopera- 
tive clinical diagnosis of COPD; clinical features of chronic chest hyperinflation and expiratory obstruction; typical features of hyperinflation on serial chest radiographs, as interpreted by an attending radiologist; evidence of airway obstruction on preoperative lung function testing, as reported by an attending pulmonologist. Prolonged mechanical ventilation was defined as mechanical ventilation via an endotracheal tube for longer than 72 hours [5].

(2) General: Mortality was defined as in-hospital mortality. Prolonged stay in the intensive care unit was defined as longer than 5 days. Stroke was defined as a persistent central neurological deficit (focal or generalized), as assessed by the study neurologist. Prolonged vasopressor exposure was defined as any requirement for a vasopressor infusion persisting longer than 72 hours. Mediastinal exploration was defined as re-exploration for bleeding within the first 24 postoperative hours. Sepsis was defined according to international guidelines as a systemic inflammatory response with a presumed or known site of infection [6,7]. A systemic inflammatory response was diagnosed by the presence of at least two of the following: body temperature alterations (hyperthermia or hypothermia); tachycardia; tachypnea; and, changes in the white blood cell count (leucocytosis or leucopenia) $[6,7]$. Pneumonia was defined as lung infection as evidenced by at least two of the following: systemic inflammatory response; chest radiograph showing alveolar infiltrative pattern compatible with pneumonia, as interpreted by an attending radiologist; and, positive sputum cultures. Sepsis and pneumonia were also combined together as a single entity, defined as infection. Donor exposures were defined as the total number of donors for blood component transfusion within the first 24 hospital hours after induction of anesthesia. Renal dysfunction was defined as a greater than $50 \%$ increase in serum creatinine with an abnormal peak serum creatinine.

The DHCA Protocol: The DHCA protocol has been extensively described [8]. Three experienced aortic surgeons were collectively responsible for over $90 \%$ of these cases in the study cohort. All patients in this cohort were cared for by a dedicated group of 10 cardiothoracic anesthesiologists. Each patient underwent balanced general endotracheal anesthesia with invasive monitoring of systemic and pulmonary arterial pressures. Temperature was continuously measured in the nasopharynx and bladder. There was continuous electroencephalographic monitoring whenever possible to guide the conduct of profound hypothermia. Transesophageal echocardiography (Multiplane 5-6.2 $\mathrm{MHz}$ probe; Sonos 5500, Philips, Bothell, WA) confirmed surgical diagnosis, guided circulatory management and assessed the surgical intervention. All patients received an antifibrinolytic, either epsilon-aminocaproic acid or aprotinin (full Hammersmith anti-inflammatory regimen), as directed by the attending anesthesiologist. Indications for aprotinin included emergency status, previous cardiac operation, and/or coagulopathy due to preoperative anticoagulant medications such as clopidogrel and warfarin.

Anticoagulation for cardiopulmonary bypass (CPB) was with bolus heparin titrated to maintain the activated clotting time $>400$ seconds, taking aprotinin exposure into account [9]. The ascending aorta was the primary site for arterial cannulation, except in acute type A dissection where the femoral artery was preferred. Venous cannulation was bicaval. During DHCA, retrograde cerebral perfusion was infused via the superior vena cava cannula. The left ventricle was vented through the right superior pulmonary vein. Balanced anterograde and retrograde cardioplegia were utilized for myocardial protection.

Patients were cooled on nonpulsatile CPB (Sarns Inc., Ann Arbor, MI) at flows for a cardiac index $>2.0 \mathrm{~L} / \mathrm{min} / \mathrm{m}^{2}$ and a mean arterial pressure $>60 \mathrm{mmHg}$. The following neuroprotective pharmacology was administered on initiation of CPB: 1g methylprednisolone, $1 \mathrm{~g}$ magnesium sulfate, 2.5 $\mathrm{mg} / \mathrm{kg}$ lidocaine, and $12.5 \mathrm{~g}$ mannitol. Alpha-stat arterial blood gas analysis was performed every thirty minutes to document the following metabolic goals: $\mathrm{pH}$ 7.35-7.45, oxygen partial pressure $>100 \mathrm{mmHg}$, carbon dioxide partial pressure $(30-40) \mathrm{mmHg}$, and hematocrit greater than $21 \%$. Cooling was continued till an isoelectric electroencephalogram or in the absence of this monitoring, for a total of 45 minutes [10].

After AAR-DHCA, the aortic graft was cannulated for antegrade arterial perfusion on CPB. Rewarming proceeded with less than a 5 degrees Celsius temperature gradient between nasopharyngeal and $\mathrm{CPB}$ perfusate temperature.

Separation from CPB was standardized. Pharmacologic circulatory support was titrated to maintain a cardiac index $>$ $2.0 \mathrm{~L} / \mathrm{min} / \mathrm{m}^{2}$ (epinephrine $2-6 \mathrm{mcg} / \mathrm{min}$ and $/$ or milrinone $0.25-0.50 \mathrm{mcg} / \mathrm{kg} / \mathrm{min})$ and mean arterial pressure $>60$ $\mathrm{mmHg}$ (phenylephrine $50-200 \mathrm{mcg} / \mathrm{min}$ and/or norepinephrine $2-8 \mathrm{mcg} / \mathrm{min}$ ). Blood component transfusion triggers were a hematocrit $<25 \%$, and/or post-protamine medical coagulopathy. All patients were managed in the intensive care unit by a dedicated intensivist.

The respiratory care after discharge from the intensive care unit was continued in the surgical ward. Patients were ambulated daily and received regular chest physiotherapy to mobilize and clear airway secretions. Patients were also taught and encouraged to utilize their incentive spirometers to assist in ongoing recovery of lung function. The respiratory therapist on the surgical ward also administered bronchodilator nebulizers as required and supervised gradual withdrawal of supplementary oxygen therapy. Perioperative pain management was aggressively maintained, including patient-controlled intravenous analgesia and patientcontrolled epidural analgesia (managed in consultation with the acute pain service from the Department of Anesthesiology and Critical Care).

Data Analysis: Cohort summary data were expressed as ratios or means with standard deviations. Standard statistical software (Stata 8, StataCorp, College Station, TX, USA) was utilized for all data analysis. Statistical significance was defined as a probability value $<0.05$. The chi-squared test was utilized to assess for significance between ratios. Students ttest was utilized to test for significance between means. Univariate analysis evaluated possible perioperative predictors for PMV after AAR-DHCA. Identified univariate predictors were subjected to multivariate analysis to yield the best predictive model for PMV after AAR-DHCA. The data analysis was supervised by the senior author (David R. Jobes MD) who has a masters degree in biostatistics. 


\section{RESULTS}

(1) Cohort Perioperative Summary (Tables 1-4): The cohort summary is presented by antifibrinolytic exposure: $66.0 \%$ aprotinin and $34.0 \%$ aminocaproic acid. The aprotinin subgroup had significantly more emergencies $(\mathrm{P}=0.009)$, a significantly lower myocardial ischemic time $(\mathrm{P}=0.04)$, and a significantly higher protamine dose $(\mathrm{P}=0.03)$. Ascending aortic and arch replacement was the most common DHCA procedure.

Table 1. Preoperative Cohort Variables

\begin{tabular}{|c|c|c|c|c|}
\hline Preoperative Variable & $\begin{array}{c}\text { Total } \\
(\mathrm{N}=144: \mathbf{1 0 0 \%})\end{array}$ & $\begin{array}{c}\text { Aprotinin } \\
(\mathrm{N}=95 / 144: 66.0 \%)\end{array}$ & $\begin{array}{l}\text { Aminocaproic Acid } \\
(\mathrm{N}=49 / 144: 34.0 \%)\end{array}$ & $\begin{array}{l}\text { Probabil- } \\
\text { ity Value }\end{array}$ \\
\hline Age (years: Mean/Standard Deviation) & $64.7 \pm 14.1$ & $64.4 \pm 14.2$ & $65.2 \pm 14.1$ & $>0.05$ \\
\hline Gender (Male/Female Ratio as \%) & $54.2 \% / 45.8 \%$ & $56.8 \% / 43.2 \%$ & $49.0 \% / 51.0 \%$ & $>0.05$ \\
\hline Body Surface Area (square meters: Mean/Standard Deviation) & $1.9 \pm 0.2$ & $1.9 \pm 0.3$ & $1.9 \pm 0.3$ & $>>0.05$ \\
\hline Emergency Cases (\%) & $40(27.8 \%)$ & $33(34.7 \%)$ & $7(14.3 \%)$ & .009 \\
\hline Reoperation Cases $(\%)$ & $27(18.8 \%)$ & $21(22.1 \%)$ & $6(12.2 \%)$ & $>>0.05$ \\
\hline Previous Stroke (\%) & $14(9.72 \%)$ & $8(8.42 \%)$ & $6(12.2 \%)$ & $>>0.05$ \\
\hline Previous Atrial Fibrillation (\%) & $15(10.4 \%)$ & $9(9.5 \%)$ & $6(12.2 \%)$ & $>>0.05$ \\
\hline History of Hypertension (\%) & $103(71.5 \%)$ & $67(70.5 \%)$ & $36(73.5 \%)$ & $>>0.05$ \\
\hline Chronic Obstructive Pulmonary Disease (\%) & $16(11.1 \%)$ & $8(8.4 \%)$ & $8(16.3 \%)$ & $>>0.05$ \\
\hline Preoperative Coumadin $(\%)$ & $26(18.1 \%)$ & $17(17.9 \%)$ & $9(18.4 \%)$ & $>>0.05$ \\
\hline Preoperative Aspirin (\%) & $83(57.6 \%)$ & $55(57.9 \%)$ & $28(57.1 \%)$ & $>>0.05$ \\
\hline Preoperative Statin (\%) & $22(15.3 \%)$ & $11(11.6 \%)$ & $11(22.4 \%)$ & $>>0.05$ \\
\hline Serum Creatinine (milligrams/deciliter: Mean/Standard Deviation) & $1.2 \pm 1.0$ & $1.2 \pm 1.2$ & $1.1 \pm 0.5$ & $>>0.05$ \\
\hline
\end{tabular}

Table 2. Cohort By Thoracic Aortic Procedure

\begin{tabular}{|c|c|c|c|}
\hline Thoracic Aortic Procedure & $\begin{array}{c}\text { Total } \\
(\mathrm{N}=144: 100 \%)\end{array}$ & $\begin{array}{c}\text { Aprotinin } \\
(\mathrm{N}=95 / 144: 66.0 \%)\end{array}$ & $\begin{array}{l}\text { Aminocaproic Acid } \\
(\mathrm{N}=49 / 144: 34.0 \%)\end{array}$ \\
\hline Aortic Arch and Ascending Aorta & $111(77.1 \%)$ & $72(75.8 \%)$ & $39(79.6 \%)$ \\
\hline Aortic Arch Only & $10(6.9 \%)$ & $7(7.3 \%)$ & $3(6.1 \%)$ \\
\hline Aortic Arch and Descending Aorta & $22(15.3 \%)$ & $15(15.8 \%)$ & $7(14.3 \%)$ \\
\hline Aortic Arch/Ascending and Descending Aorta & $1(0.7 \%)$ & $1(1.1 \%)$ & $0(0 \%)$ \\
\hline
\end{tabular}

Table 3. Intraoperative Cohort Variables

\begin{tabular}{|c|c|c|c|c|}
\hline Intraoperative Variable & $\begin{array}{c}\text { Total } \\
\text { (N=144: } 100 \%) \\
\text { Mean/ } \\
\text { Standard Deviation }\end{array}$ & $\begin{array}{c}\text { Aprotinin } \\
\text { (N=95/144: } 66.0 \%) \\
\text { Mean/ } \\
\text { Standard Deviation }\end{array}$ & $\begin{array}{c}\text { Aminocaproic Acid } \\
(\mathrm{N}=49 / 144: 34.0 \%) \\
\text { Mean/Standard Deviation }\end{array}$ & $\begin{array}{c}\text { Probability } \\
\text { Value }\end{array}$ \\
\hline Cardiopulmonary Bypass Time (minutes) & $196 \pm 43.5$ & $196 \pm 44.0$ & $196 \pm 42.9$ & $>0.05$ \\
\hline Myocardial Ischemia Time (minutes) & $132 \pm 45.8$ & $127 \pm 48.8$ & $141 \pm 38.5$ & .04 \\
\hline Deep Hypothermic Circulatory Arrest Time (minutes) & $37.9 \pm 15.0$ & $38.9 \pm 14.9$ & $36.0 \pm 15.3$ & $>0.05$ \\
\hline Temperature Nadir (degrees Celsius) & $14.2 \pm 2.4$ & $14.1 \pm 2.1$ & $14.3 \pm 2.8$ & $>0.05$ \\
\hline Fentanyl (micrograms per kilogram) & $39.2 \pm 17.7$ & $38.7 \pm 18.5$ & $40.3 \pm 16.2$ & $>0.05$ \\
\hline Midazolam (milligrams per kilogram) & $.26 \pm .17$ & $.24 \pm .08$ & $.29 \pm .26$ & $>0.05$ \\
\hline Pancuronium (milligrams per kilogram) & $.15 \pm .08$ & $.14 \pm .07$ & $.17 \pm .08$ & $>0.05$ \\
\hline Baseline Activated Clotting Time (seconds) & $144.6 \pm 51.7$ & $144 \pm 38.9$ & $145 \pm 69.5$ & $>0.05$ \\
\hline Maximum Activated Clotting Time (seconds) & $887 \pm 205$ & $907 \pm 203$ & $848 \pm 206$ & $>0.05$ \\
\hline Total Heparin Dose (units) & $29649 \pm 11983$ & $30779 \pm 11724$ & $27459 \pm 12295$ & $>0.05$ \\
\hline Total Protamine Dose (milligrams) & $170 \pm 69.6$ & $178 \pm 72.3$ & $155 \pm 62.0$ & .03 \\
\hline
\end{tabular}


Table 4A. Major Clinical Outcomes

\begin{tabular}{|c|c|c|c|c|}
\hline Major Clinical Outcome & $\begin{array}{c}\text { Total } \\
(\mathrm{N}=144: 100 \%)\end{array}$ & $\begin{array}{c}\text { Aprotinin } \\
(\mathrm{N}=95 / 144: 66.0 \%)\end{array}$ & $\begin{array}{l}\text { Aminocaproic Acid } \\
(\mathrm{N}=49 / 144: 34.0 \%)\end{array}$ & $\begin{array}{c}\text { Probability } \\
\text { Value }\end{array}$ \\
\hline Mortality & $16(11.1 \%)$ & $11(11.6 \%)$ & $5(10.2 \%)$ & $>0.05$ \\
\hline Length of Stay in Intensive Care Unit (days) & $6.7 \pm 10.3$ & $7.8 \pm 11.5$ & $4.6 \pm 6.8$ & .02 \\
\hline Atrial Fibrillation & $49(34.0 \%)$ & $30(31.6 \%)$ & $19(38.8 \%)$ & $>0.05$ \\
\hline Stroke & $12(8.3 \%)$ & $10(10.5 \%)$ & $2(4.1 \%)$ & $>0.05$ \\
\hline Prolonged Vasopressor Dependence & $15(10.4 \%)$ & $12(12.6 \%)$ & $3(6.12 \%)$ & $>0.05$ \\
\hline Mediastinal Exploration within 24 Hours & $5(3.5 \%)$ & $3(3.2 \%)$ & $2(4.1 \%)$ & $>0.05$ \\
\hline Sepsis & $8(5.56 \%)$ & $5(5.3 \%)$ & $3(6.1 \%)$ & $>0.05$ \\
\hline Renal Dysfunction & $33(22.9 \%)$ & $28(29.5 \%)$ & $5(10.2 \%)$ & .003 \\
\hline
\end{tabular}

Table 4B. Pulmonary Outcomes

\begin{tabular}{|c|c|c|c|c|}
\hline Pulmonary Outcome & $\begin{array}{c}\text { Total } \\
(\mathrm{N}=144: \mathbf{1 0 0} \%)\end{array}$ & $\begin{array}{c}\text { Aprotinin } \\
(\mathrm{N}=95 / 144: 66.0 \%)\end{array}$ & $\begin{array}{l}\text { Aminocaproic Acid } \\
(\mathrm{N}=49 / 144: 34.0 \%)\end{array}$ & $\begin{array}{l}\text { Probability } \\
\text { Value }\end{array}$ \\
\hline Prolonged Mechanical Ventilation & $31(21.5 \%)$ & $24(25.3 \%)$ & $7(14.3 \%)$ & .09 \\
\hline Pneumonia & $11(7.64 \%)$ & $7(7.37 \%)$ & $4(8.16 \%)$ & .56 \\
\hline Tracheal Re-intubation & $25(17.4 \%)$ & $19(20.0 \%)$ & $6(12.2 \%)$ & .18 \\
\hline Tracheostomy Required & $18(12.5 \%)$ & $16(16.8 \%)$ & $2(4.08 \%)$ & .02 \\
\hline
\end{tabular}

Further perioperative outcomes between antifibrinolytic subgroups were similar except for length of ICU stay and renal dysfunction. These outcome differences have been addressed in prior publications $[11,12]$.

The incidence of PMV was $21.5 \%$, with no significant relationship to aprotinin exposure. Further pulmonary outcomes are detailed in Table 4B: the tracheostomy rate was significantly higher in the aprotinin subgroup.

(2) Multivariate Predictors of Mortality and Prolonged ICU Stay (Tables 5,6): Multivariate predictors for mortality were sepsis, stroke, and mediastinal exploration within 24 hours [13]. Multivariate predictors for prolonged ICU stay were stroke, prolonged vasopressor dependence, and renal dysfunction [11]. Prolonged mechanical ventilation was not an independent multivariate predictor of mortality or prolonged ICU stay after AAR-DHCA.

Table 5. Multivariate Predictors for Mortality

\begin{tabular}{|l|c|}
\hline \multicolumn{1}{|c|}{ Multivariate Predictor } & $\begin{array}{c}\text { Odds Ratio/ } \\
\text { Confidence Interval/ } \\
\text { P-Value }\end{array}$ \\
\hline \hline Sepsis & $21.3: 1 / 3.8-121 / 0.001$ \\
\hline Stroke & $7.4: 1 / 1.9-28.7 / 0.004$ \\
\hline Mediastinal Exploration within 24 Hours & $7.7: 1 / 1.3-45.1 / 0.02$ \\
\hline
\end{tabular}

(3) Multivariate Predictors of Prolonged Mechanical Ventilation (Table 7,8): Candidate PMV predictors were statistically evaluated in univariate analysis. The identified univariate predictors were then subjected to multivariate analysis to yield the following clinical predictors for PMV
Table 6. Multivariate Predictors for Prolonged Stay in the Intensive Care Unit

\begin{tabular}{|l|c|}
\hline \multicolumn{1}{|c|}{ Multivariate Predictor } & $\begin{array}{c}\text { Odds Ratio/ } \\
\text { Confidence Interval/ } \\
\text { P-Value }\end{array}$ \\
\hline \hline Stroke & $15.1: 1 / 3.6-64.0 / 0.001$ \\
\hline Prolonged Vasopressor Dependence & $7.3: 1 / 1.3-41.3 / 0.024$ \\
\hline Renal Dysfunction & $4.8: 1 / 1.7-13.6 / 0.003$ \\
\hline
\end{tabular}

Table 7. Univariate Predictors for Prolonged Mechanical Ventilation

\begin{tabular}{|c|c|}
\hline Univariate Predictor & $\begin{array}{c}\text { Odds Ratio/ } \\
\text { Confidence Interval/ } \\
\text { P-Value }\end{array}$ \\
\hline \hline DHCA Duration & $1.04: 1 / 1.01-1.07 / 0.003$ \\
\hline Packed Red Blood Cell Transfusion & $1.26: 1 / 1.1-1.5 / 0.002$ \\
\hline Stroke & $6.25: 1 / 2.2-17.7 / 0.001$ \\
\hline Renal Dysfunction & $11.0: 1 / 4.3-28.4 /<0.0001$ \\
\hline Chronic Obstructive Pulmonary Disease & $13.3: 1 / 2.54-69.9 / 0.002$ \\
\hline Infection & $45.7: 1 / 9.50-219 / 0.0001$ \\
\hline
\end{tabular}

after AAR - DHCA: chronic obstructive pulmonary disease; stroke; and, infection.

\section{DISCUSSION}

This study confirms that PMV after AAR-DHCA remains common with an incidence of $21.5 \%$. This incidence, 
however, was in the reported range [1-3]. However, series from high-volume thoracic aortic centers focused predominantly on descending thoracic aortic procedures with or without DHCA $[1-3,5,14]$. These procedures typically involve major thoracotomy (as compared to sternotomy) and extensive diaphragmatic dissection, In contrast, this clinical study focuses on all aortic arch repairs in which $84 \%$ were via sternotomy. Adult AAR-DHCA has a significant incidence of PMV, regardless of surgical incision. The explanation for this observation lies in the multivariate predictors for PMV which will be discussed later in this section.

Table 8. Multivariate Predictors for Prolonged Mechanical Ventilation

\begin{tabular}{|l|c|}
\hline \multicolumn{1}{|c|}{ Multivariate Predictor } & $\begin{array}{c}\text { Odds Ratio/ } \\
\text { Confidence Interval/ } \\
\text { P-Value }\end{array}$ \\
\hline \hline Stroke & $4.56: 1 / 1.3-16.7 / 0.022$ \\
\hline Chronic Obstructive Pulmonary Disease & $12.1: 1 / 1.8-80.1 / 0.009$ \\
\hline Infection & $33.7: 1 / 6.6-172 /<0.0001$ \\
\hline
\end{tabular}

The identified multivariate predictors for mortality and prolonged ICU stay in this study do not include PMV, as reported previously [2]. Closer inspection explains this paradox. The mechanisms of all three outcomes (mortality; prolonged ICU stay; PMV) overlap significantly with respect to stroke and sepsis. Thus, PMV results from stroke and sepsis which are also independent predictors for mortality and prolonged ICU stay in the adult AAR-DHCA population.

It must be remembered, however, that mechanical ventilation itself does predispose to lung injury due to ventilatorassociated pneumonia and pulmonary inflammation [15-17]. With respect to lung stretch and inflammation, conventional mechanical ventilation strategies were employed in this study: tidal volumes were $8-10 \mathrm{ml} / \mathrm{kg}$, and positive endexpiratory pressure was frequently utilized $(5-7.5 \mathrm{~cm}$ water $)$ [14]. In this study, clinical pneumonia only became a multivariate predictor when combined with sepsis as infection. Furthermore, in regard to ventilator-associated pulmonary inflammation, modulators of the inflammatory response such as aprotinin, statins and systemic steroids will have to be considered in future trial design. On the basis of this clinical trial, the precise contribution of these pathophysiologic mechanisms cannot be fully quantified. Further study of PMV after AAR-DHCA is required to fully delineate whether these interactions achieve clinical significance.

The identified multivariate predictors for PMV make sense. Chronic pulmonary obstructive disease is a commonly present in the AAR-DHCA population and represents a significant etiology of decreased pulmonary reserve. Therefore, perioperative injury is more likely to result in clinical dysfunction, namely PMV. This finding of the predictive power of decreased pulmonary reserve is consistent with PMV in major noncardiac surgery, as well as cardiac and thoracic aortic surgery $[2,3,18,19]$.

The remaining multivariate predictors, stroke and infection, represent perioperative opportunities for intervention. They have discussed in prior publications about their role in mortality and prolonged ICU stay after AAR-DHCA. ${ }^{11-12}$
Stroke prolongs mechanical ventilation due to the consequent interference with adequate protection and maintenance of the airway (e.g. impaired cough; swallowing dysfunction with aspiration risk). Infection, especially pneumonia, prolongs mechanical ventilation due to decreased functional pulmonary parenchyma. A full discussion of prevention and risk reduction for these 2 perioperative complications after AAR-DHCA is beyond the scope of this article. Their correlation with PMV adds further priority to focus perioperative intervention on the protection against brain injury and infection after AAR-DHCA.

What about the relationship of aprotinin to PMV after AAR - DHCA? Due to its anti-inflammatory properties, aprotinin could protect against lung injury by modulating the inflammatory response to cardiopulmonary bypass. Although randomized trials in pediatric and adult cardiac surgery have shown a pulmonary protective effect of aprotinin, it has not been a consistent observation in meta-analysis [20-23]. A recent retrospective clinical trial in adult thoracic aortic surgery detected a significant association between aprotinin exposure and reduced pulmonary complications [24]. The cohort in this study was only $66.1 \%$ AAR-DHCA [24, 25].

In the present study, the cohort is $100 \%$ AAR-DHCA, but aprotinin, just as in the aforementioned trial, was not randomized. In multivariate analysis, aprotinin exposure was not associated with PMV. However, aprotinin exposure was significantly more common in the emergency cases which were significantly more likely to have prolonged stay in the intensive care unit, and require tracheostomy. Clearly, on the basis of these results confounded by association, further study is required to conclusively answer this question. Ideally, it would be a randomized trial of aprotinin in AARDHCA, adequately powered to detect a clinically meaningful reduction in PMV. Given the withdrawal of aprotinin from the world market recently due to safety concerns, this further evaluation of aprotinin in adult AAR-DHCA is on hold till the safety issues are resolved [26, 27].

\section{LIMITATIONS OF THIS STUDY}

Firstly, this study is retrospective and so can only detect association but not causality. Secondly, aprotinin exposure was not randomized. Thirdly, this AAR-DHCA cohort consists of surgically distinct subgroups that may have different levels of risk for PMV after AAR-DHCA. These limitations must be taken into account when considering the main findings of the study.

\section{CONCLUSION}

Prolonged mechanical ventilation after AAR-DHCA is common, but does not independently predict mortality or ICU stay. Chronic obstructive pulmonary disease, stroke and infection are independent predictors of PMV after AARDHCA. Perioperative intervention should focus on protection against stroke and infection.

\section{ACKNOWLEDGEMENTS}

Study Support: Department of Anesthesiology and Critical Care, Hospital of the University of Pennsylvania. 


\section{REFERENCES}

[1] Augoustides JG, Floyd TF, McGarvey M, et al. Major clinical outcomes in adults undergoing thoracic aortic surgery requiring deep hypothermic circulatory arrest: quantification of organ-based perioperative outcome and detection of opportunities for perioperative intervention. J Cardiothorac Vasc Anesth 2005; 19: 446-52.

[2] Etz CD, Di Luozzo G, Bello R, et al. Pulmonary complications after descending thoracic and thoracoabdominal aortic aneurysm repairs. Ann Thorac Surg 2007; 83: S870-876.

[3] Safi HJ, Miller CC $3^{\text {rd }}$, Subramanjam MH, et al. Thoracic and thoracoabdominal aneurysm repair using cardiopulmonary bypass, profound hypothermia and circulatory arrest via left side of chest incision. J Vasc Surg 1998; 28: 591-8.

[4] Sedrakyan A, Wu A, Sedrakyan G, et al. Aprotinin use in thoracic aortic surgery: safety and outcomes. J Thorac Cardiovasc Surg 2006; 132: 909-917.

[5] Engle J, Safi HJ, Miller CC $3^{\text {rd }}$, et al. The impact of diaphragm management on prolonged ventilator support after thoracoabdominal aortic repair. J Vasc Surg 1999; 29: 150-6.

[6] Larosa SP. Sepsis: menu of new approaches replaces one therapy for all. Cleve Clin J Med 2002; 69: 65-73

[7] Bone RC, Balk RA, Cerra FB, et al. Definitions for sepsis and organ failure and guidelines for the use of innovative therapies in sepsis. The ACCP/SCCM Consensus Conference Committee. American College of Chest Physicians/Society of Critical Care Medicine. Chest 1992; 101: 1644-55.

[8] Appoo JJ, Augoustides JG, Pochettino A, et al. Perioperative outcome in adults undergoing elective deep hypothermic circulatory arrest with retrograde cerebral perfusion in proximal aortic arch repair: evaluation of protocol-based care. J Cardiothorac Vasc Anesth 2006; 20: 3-7.

[9] Dietrich W, Dilthey G, Spannagl M, et al. Influence of high-dose aprotinin on anticoagulation, heparin requirement, and celite- and kaolin-activated clotting time in heparin-pretreated patients undergoing open-heart surgery. A double-blind, placebo-controlled study. Anesthesiology 1995; 83:679-89.

[10] Stecker MM, Cheung AT, Pochettino A, et al. Deep hypothermic circulatory arrest: effects of cooling on the electroencephalogram and evoked potentials. Ann Thorac Surg 2001; 71: 14-21.

[11] Augoustides JG, Pochettino A, Ochroch EA, et al. Clinical predictors for prolonged intensive care unit care stay in adults undergoing thoracic aortic surgery requiring deep hypothermic circulatory arrest. J Cardiothorac Vasc Anesth 2006; 20: 8-13.

[12] Augoustides JG, Pochettino A, Ochroch EA, et al. Renal dysfunction after thoracic aortic grafting requiring deep hypothermic circulatory arrest: definition, incidence, and clinical predictors. J Cardiothorac Vasc Anesth 2006; 20: 673-7.
[13] Augoustides JG, Pochettino A, McGarvey M, et al. Clinical predictors for mortality in adults undergoing thoracic aortic surgery requiring deep hypothermic circulatory arrest. Ann Card Anaesth 2006; 9: 114-19.

[14] Svensson LG, Hess KR, Coselli JS, et al. A prospective study of respiratory failure after high-risk surgery on the thoracoabdominal aorta. J Vasc Surg 1991; 14: 271-82.

[15] Klompas M. Does this patient have ventilator-associated pneumonia? JAMA 2007; 297: 1583-93.

[16] Miranda DR, Gommers D, Papadakos PJ, et al. Mechanical ventilation affects pulmonary inflammation in cardiac surgery patients the role of the open-lung concept. J Cardiothorac Vasc Anesth 2007; 21: 279-84.

[17] Reis Miranda D, Gommers D, Struijs A, et al. Ventilation according to the open lung concept attenuates pulmonary inflammatory response in cardiac surgery. Eur J Cardiothorac Surg 2005; 28: 889-95

[18] Arozullah Am, Daley J, Henderson WG, et al. Multifactorial risk index for predicting postoperative respiratory failure in men after major noncardiac surgery. Ann Surg 2000; 232: 242-53.

[19] Branca P, Mcgaw P, Light R. Factors associated with prolonged mechanical ventilation following coronary artery bypass surgery. Chest 2001; 119: 537-46.

[20] Mossinger H, Dietrich W, Braun SL, et al. High-dose aprotinin reduces activation of hemostasis, allogeneic blood requirement, and duration of postoperative ventilation in pediatric cardiac surgery. Ann Thorac Surg 2003; 75: 430-37.

[21] Erdogan M, Kalaycloglu S, Iriz E. Protective effect of aprotinin against lung damage in patients undergoing CABG surgery. Acta Cardiol 2005; 60: 367-72.

[22] Sedrakyan A, Treasure T, Elefteriades JA. Effect of aprotinin on clinical outcomes in coronary artery bypass graft surgery: a systematic review and meta-analysis of randomized clinical trials. $\mathrm{J}$ Thorac Cardiovasc Surg 2004; 128: 442-48.

[23] Sedrakyan A. Improving clinical outcomes in coronary artery bypass graft surgery. Am J Health Syst Pharm 2005; 62: S19-S23.

[24] Sedrakyan A, Wu A, Sedrakyan G, et al. Aprotinin in thoracic aortic surgery: safety and outcomes. J Thorac Cardiovasc Surg 2006; 132: 909-17.

[25] Augoustides JG. Limitations with aprotinin in thoracic aortic bleeding: understanding the clinical outcome beyond bleeding. J Thorac Cardiovasc Surg 2007; 134: 269.

[26] Strouch ZY, Chaney MA, Augoustides JG, Spiess BD. Case 12008. One institution's decreasing use of aprotinin during cardiac surgery in 2006. J Cardiothorac Vasc Anesth 2008; 22: 139-46.

[27] Augoustides JG. Con: Aprotinin should not be used in cardiac surgery with cardiopulmonary bypass. J Cardiothorac Vasc Anesth 2007; 21: 302-04 